\title{
Medium-term Anatomical Results of Laser Peripheral Iridoplasty: An Anterior Segment Optical Coherence Tomography Study
}

\author{
1Joobin Hooshmand, ${ }^{2}$ James CY Leong, ${ }^{3}$ Jeremy O'Connor, ${ }^{4}$ Ghee S Ang, ${ }^{5}$ Anthony P Wells
}

\begin{abstract}
Aim: To evaluate by anterior segment optical coherence tomography (AS-OCT) the medium-term (mean duration 3.2 years) anatomical changes in the anterior chamber angle (ACA) after laser peripheral iridoplasty.
\end{abstract}

Materials and methods: This is a longitudinal, retrospective case series of 31 eyes of 31 patients with primary angle-closure suspicion, primary angle closure (PAC), or primary angleclosure glaucoma (PACG) who underwent laser peripheral iridoplasty. All patients had persistent iridotrabecular contact (ITC) despite the presence of a patent peripheral iridotomy (PI). An AS-OCT was performed in dark conditions before and after laser iridoplasty. Quadrants of ITC, intraocular pressure (IOP), and the AS-OCT parameters of the temporal and nasal ACAs were measured and analyzed.

Results: Prior to iridoplasty, the average number of quadrants of ITC was 3.3. At the first postiridoplasty visit (mean duration 6.8 weeks), this reduced to 1.7 quadrants but increased to 1.9 by the final follow-up visit (mean duration 3.2 years). Twentyfive patients $(80.1 \%)$ had less ITC at the first postlaser visit increasing to $27(87.1 \%)$ patients by the final visit. Two $(6.5 \%)$ required a second iridoplasty, while $3(9.7 \%)$ required cataract surgery. All parameters of angle width showed a statistically significant increase in magnitude. All patients maintained IOP $\leq 21 \mathrm{~mm} \mathrm{Hg}$ throughout the follow-up period.

Conclusion: Iridoplasty is a useful adjunct in widening the ACA, particularly in those with persistent angle closure after iridotomy but with no cataract. While not successful in all patients, it can act as a temporizing measure to widen the drainage angle until such time that cataract surgery can be performed.

Clinical significance: Laser peripheral iridoplasty can be used as an adjunct in angle-closure glaucoma patients with no cataract.

\footnotetext{
1,4,5 Consultant, ${ }^{2}$ Registrar, ${ }^{3}$ Fellow

1,3,4 Department of Ophthalmology, Royal Victorian Eye and Ear Hospital, East Melbourne, Victoria, Australia

${ }^{2}$ Department of Ophthalmology, Capital \& Coast District Health Board, Wellington, New Zealand

${ }^{5}$ Department of Ophthalmology, Capital Eye Specialists Wellington, New Zealand

Corresponding Author: Joobin Hooshmand, Consultant Department of Ophthalmology, Royal Victorian Eye and Ear Hospital, East Melbourne, Victoria, Australia, Phone: +61401689977, e-mail: joobin.hooshmand@gmail.com
}

Keywords: Iridoplasty, Longitudinal, Optical coherence tomography, Primary angle-closure glaucoma.

How to cite this article: Hooshmand J, Leong JCY, O'Connor J, Ang GS, Wells AP. Medium-term Anatomical Results of Laser Peripheral Iridoplasty: An Anterior Segment Optical Coherence Tomography Study. J Curr Glaucoma Pract 2017;11(3):113-119.

Source of support: Capital Vision Research Trust, Wellington, New Zealand.

\section{Conflict of interest: None}

\section{INTRODUCTION}

Glaucoma is the leading cause of irreversible blindness worldwide. ${ }^{1}$ Despite its lower prevalence in comparison with primary open-angle glaucoma, PACG is responsible for a disproportionately large share of the visual morbidity attributable to glaucoma in Asian populations. ${ }^{2}$ The PACG is also a significant, and likely underdiagnosed, condition in Caucasian populations. ${ }^{3}$

Foster et $\mathrm{al}^{4}$ described standardized definitions of PACG along a spectrum of increasing severity from primary angle-closure suspect (PACS), PAC, and PACG. ${ }^{5}$ In the setting of PAC, the recommended initial nonsurgical means of widening the ACA is by laser PI, which eliminates relative pupillary block. ${ }^{5}$ However, a proportion of eyes will still have residual angle closure, despite a successfully performed and patent iridotomy. ${ }^{6}$ In these eyes, particularly if they have no cataract, laser peripheral iridoplasty may be useful to treat persistent appositional angle closure occurring through non pupillary block mechanisms. ${ }^{7,8}$ Iridoplasty is thought to widen the ACA by thermal-induced contraction of the peripheral iris, as well as cross-sectional thinning of iris tissue. ${ }^{9}$ Current evidence suggests that iridoplasty is a useful adjunctive treatment tool for angle closure. ${ }^{10}$

Gonioscopy is the gold standard for assessment of ACA, but it can be highly subjective and observer dependent. ${ }^{11}$ Anterior segment optical coherence tomography allows objective, precise, and reproducible quantification of various anterior segment and angle anatomy parameters. $^{12-14}$ The AS-OCT is a noncontact imaging modality that rapidly obtains high-resolution cross-sectional images of the anterior segment with the patient seated 
upright. These features are advantageous compared with older imaging modalities, such as ultrasound biomicroscopy. ${ }^{15}$ Previous work by our group utilized AS-OCT to outline changes in anatomical features of the ACA in the short term following iridoplasty in a cohort of patients with persistent angle closure despite a patent iridotomy. ${ }^{16}$ Apart from a case series utilizing gonioscopy, ${ }^{7}$ there is a paucity of quantitative descriptions of the medium-term anatomical results of iridoplasty in the published literature. The aim of this study was to use AS-OCT to quantify the medium-term (mean duration 3.2 years) changes in ACA anatomy after iridoplasty in patients with residual angle closure despite a patent iridotomy.

\section{MATERIALS AND METHODS}

This was a retrospective case series of 31 patients who underwent diode iridoplasty at Capital Eye Specialists, Wellington, New Zealand, recruited over a 41-month interval. All patients in this cohort had previously undergone laser iridotomy for PAC, PACS, or PACG, and had subsequent iridoplasty for persistent ITC despite a patent iridotomy.

Each newly referred patient with suspected angle closure received a full ocular examination. This included best-corrected Snellen visual acuity, slit-lamp evaluation, Goldmann applanation tonometry, corneal pachymetry, undilated fundoscopy, gonioscopy, and time domain AS-OCT imaging. The AS-OCT was performed by ophthalmic imaging technicians with the slit-lamp OCT (Heidelberg Engineering, GmBH, Dossenheim, Germany) in both uniform light and the dark with all room lights switched off. The scans were centered on the pupil.

The glaucoma specialist (A.P.W.) made the clinical decision as to whether the iridotomy was indicated based on AS-OCT and gonioscopy findings. The gonioscopy threshold for iridotomy was nonvisibility of the trabecular meshwork in at least $180^{\circ}$ of the ACA, consistent with the Association of International Glaucoma Societies consensus on angle-closure gonioscopy criteria. ${ }^{17}$ The AS-OCT threshold was extrapolated from these criteria as being the presence of ITC, visualized as apposition of peripheral iris to the inner corneoscleral wall anterior to the scleral spur, in at least two of four quadrants in dark conditions. In our study, this was defined to be consistent with PACS. The additional presence of peripheral anterior synechiae and/or IOP > $21 \mathrm{~mm} \mathrm{Hg}$ distinguished PAC. The diagnosis of PACG was made if there were concurrent optic disk and visual field changes characteristic of glaucoma.

Laser PI was performed using the ophthalmic neodymium-doped yttrium-aluminum-garnet laser (Laserex Tango Nd:YAG, Ellex Medical, Australia) and an Abraham iridotomy contact lens. The PI was placed superiorly as close to the vertical meridian and as far peripherally as practical. Full-thickness perforation was confirmed by the gush of pigment and aqueous fluid from the posterior chamber into the anterior chamber. All iridotomies were confirmed patent in the study population.

The postiridotomy eyes with residual ITC in two or more quadrants in dark conditions as confirmed on repeat AS-OCT were deemed to still have occludable angles and were selected for iridoplasty. The mean time from iridotomy to iridoplasty was 34 weeks. Iridoplasty was performed using the Oculight SLx diode laser. Pupils were constricted with pilocarpine $2 \%$. Thirty to thirtyfive laser shots were applied on the iris as peripherally as possible over $360^{\circ}$, using a power between 200 and $350 \mathrm{~mW}$. Maximum treatment time was 2.5 seconds, and spot size was $500 \mu \mathrm{m}$. The power and duration were titrated to be just enough to cause iris contraction but not superficial iris charring. After iridoplasty, patients were prescribed 5 days of topical prednisolone acetate $1.0 \%$ to relieve postlaser inflammation.

All patients received regular AS-OCT as well as routine clinical examination which included IOP measurement and gonioscopy at their follow-up visits. These visits were generally scheduled 1 to 2 months after iridoplasty and every 6 months thereafter. The AS-OCT images from the final visit were reviewed and analyzed for each patient and compared with the images taken prior to iridoplasty. Although multiple scans of all four ACA quadrants were captured and treatment decisions were based on images from all four quadrants, only the horizontal images were analyzed in this study because these provided a clearer view of all ACA structures, in particular the scleral spur and peripheral iris recess, compared with the vertical images.

A single observer, a glaucoma fellow (G.S.A.), who was masked to the identity and sequence of the images being evaluated, measured the nasal and temporal quadrants for all eyes. The observer selected the best-quality image with clearly identifiable anatomical landmarks from a series of images of the nasal and temporal quadrants all centered on the pupil. Following the selection of the location of the scleral spur and iris recess apex, various anterior chamber drainage angle parameters were calculated using the slit-lamp OCT's inbuilt analysis software including trabecular-iris angle (TIA), angle opening distance (AOD), trabecular-iris space area (TISA), and anterior chamber depth. Further parameters not included in the in-built analysis software were measured including trabecular-iris contact length (TICL), iris thickness (IT), and maximum iris bow height (MIBH). We had previously demonstrated moderate-to-good intraobserver reproducibility with the intraclass correlation coefficient 

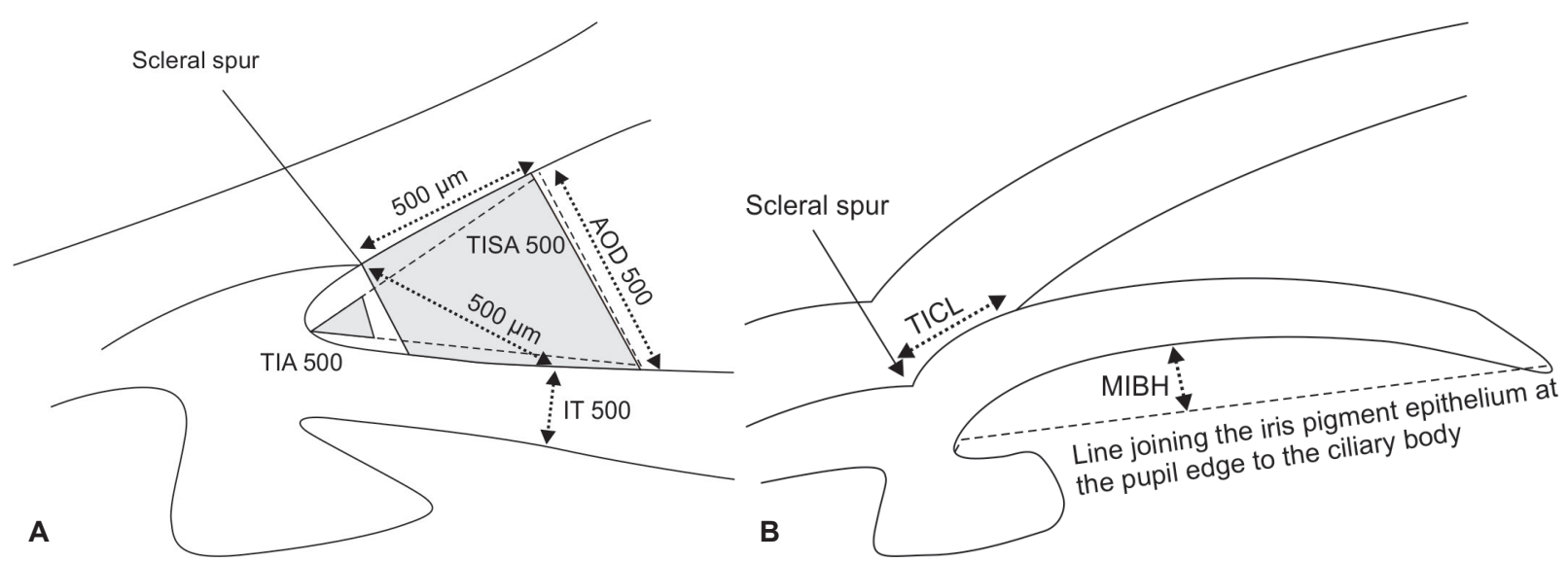

Figs $1 \mathrm{~A}$ and $\mathrm{B}$ : Anterior chamber drainage angle parameters measured with AS-OCT

statistic on all of these AS-OCT parameters in a larger cohort of patients using the same AS-OCT instrument and analysis software..$^{18}$ Although the AS-OCT images were captured in both light and dark conditions, only results of scans in the dark were used for analysis.

Figure 1 summarizes the parameters measured with AS-OCT. The AOD 500 and 750 were the perpendicular distances from the trabecular meshwork at 500 and $750 \mu \mathrm{m}$ anterior to the scleral spur to the anterior iris surface. ${ }^{19}$ The TIA 500 was considered the angle between the point of the trabecular meshwork $500 \mu \mathrm{m}$ from the scleral spur and the point on the anterior iris perpendicularly, with the apex at the iris recess. ${ }^{19}$ The TIA 750 was similar to TIA 500 with the exception of the angle measured from the point of the trabecular meshwork $750 \mu \mathrm{m}$ from the scleral spur. The TISA 500 was the trapezoidal area bordered anteriorly by the AOD 500, posteriorly by a line from the scleral spur perpendicular to the plane of the inner sclera to the anterior iris, superiorly by the inner corneoscleral wall, and inferiorly by the anterior iris surface. ${ }^{20}$ The TISA 750 was similar to TISA 500, except that it was bordered anteriorly by the AOD 750. The IT 500 was the perpendicular distance from the anterior iris surface at $500 \mu \mathrm{m}$ from the scleral spur to the posterior iris pigment epithelial surface. ${ }^{21}$ The TICL was the length of contact between the anterior iris surface and the inner corneoscleral wall. ${ }^{20}$ The MIBH was used as a surrogate marker for iris curvature and was the perpendicular distance measured from the posterior iris pigment epithelial surface at its apex (the point where iris bowing was at its greatest) to the line joining the iris pigment epithelium at the pupil edge to its insertion at the ciliary body. ${ }^{22}$ The hyperreflective curve on the posterior iris surface marked the iris pigment epithelium; its insertion at the ciliary body was the point where the hyperreflective curve terminated within the ciliary body. Intraocular pressure was measured as an additional outcome.
Iridoplasty was defined as successful when ITC was observed in maximum of one quadrant, partially successful if ITC was observed in maximum of two quadrants, and failure if no improvement in ITC was observed and/ or cataract extraction was required to treat angle closure.

Data were analyzed using Microsoft Excel software. Basic descriptive statistics was conducted for patient demographics. Comparison of means was performed with the paired t-test for parametric data, while the Wilcoxon signed-rank test was used for nonparametric data. A p-value less than 0.05 was considered to be statistically significant. The study was conducted according to the tenets of the Declaration of Helsinki and had received approval from the Central Regional Ethics Committee of New Zealand.

Exclusion criteria were secondary angle closure, such as from uveitis, angle neovascularization and intumescent cataract, previous trauma, previous intraocular surgery, or poor-quality AS-OCT images that were unsuitable for angle evaluation. Only the right eye was used for analysis if both eyes were eligible.

\section{RESULTS}

A total of 31 eyes of 31 patients were included in this study. The mean age at treatment with iridoplasty was $56.2( \pm 9.6)$ years. Twenty-nine patients $(94 \%)$ were female. The majority of the patients were Caucasian $(90 \%)$, with 2 being of Asian and 1 of Maori background. Four patients (13\%) had PACG. The mean duration of follow-up after iridoplasty to the final follow-up visit was 38.6 months $/ 3.2$ years [2.4-71.2 months; standard deviation (SD) 20.5]. Four patients (12.9\%) underwent cataract surgery, at a mean of 24 months postiridoplasty, and $3(9.7 \%)$ due to persistent angle closure postiridoplasty. Two patients (6.5\%) required redo-iridoplasty for persistent residual ITC, at a mean of 40 weeks postinitial Iridoplasty. All patients maintained IOP less than or equal to 21 at all follow-up clinic visits. Only the patients with confirmed 
glaucomatous damage (3 eyes) were on glaucoma treatment drops and only with a maximum of two agents.

Prior to iridoplasty, the average number of quadrants of ITC was 3.3. At the first postiridoplasty visit (mean duration 6.8 weeks), this reduced to 1.7 quadrants but increased to 1.9 by the final follow-up visit (mean duration 3.2 years). Twenty-five patients $(80.1 \%$ ) had less ITC at the first postlaser visit, increasing to 27 patients (87.1\%) by the final visit.

At the first follow-up visit, the number of successful iridoplasties was 24 (77.4\%), with 14 (45.1\%) considered complete success and $10(32.3 \%)$ partial success. This number changed to $8(25.8 \%)$ completely successful iridoplasties and 18 (58.1\%) partially successful cases by the end of the follow-up period. Overall 26 patients $(83.9 \%)$ were successfully treated with iridoplasty at the final follow-up visit.

After iridoplasty, all indicators of angle width (AOD 500, AOD 750, TIA 500, TIA 750, and TISA 500) showed a statistically significant increase in magnitude over the medium term. The IT 500 showed a statistically significant reduction in IT postiridoplasty. The TICL and MIBH both showed a statistically significant reduction in nasal and temporal angles. These parameters are summarized in Table 1. Typical AS-OCT changes pre- and postiridoplasty are shown in Figure 2. Typical postiridoplasty scar appearance is shown in Figure 3.

\section{DISCUSSION}

Numerous AS-OCT studies have confirmed the changes in the ACA that occur after iridotomy. ${ }^{18,23-25}$ In the setting of persistent occludable angles after iridotomy, we previously documented that in the short term, additional iridoplasty brings about significant widening of the ACA. ${ }^{16}$ This study does not report on gonioscopic findings as the aim was to quantify changes in angle configuration using AS-OCT.

We defined success as "complete" when ITC was observed in a maximum of one quadrant in dark conditions on AS-OCT, and hence, the eye was considered nonoccludable. A total of eight patients (25.8\%) achieved complete success and were considered nonoccludable at their final follow-up visit. "Partial success" was when iridoplasty was able to reduce the number of quadrants

Table 1: Changes in the anterior chamber and angle parameters postiridoplasty in the medium term

\begin{tabular}{|c|c|c|c|c|}
\hline Parameter & Mean preiridoplasty (SD) & Mean postiridoplasty (SD), $\mu m$ & Mean diff (95\% Cl) & $p$-value* \\
\hline \multicolumn{5}{|l|}{ AOD $500, \mu \mathrm{m}$} \\
\hline Temporal & $77(79)$ & $175(92)$ & $97(64-130)$ & $<0.0001$ \\
\hline Nasal & $56(61)$ & $151(74)$ & $95(63-127)$ & $<0.0001$ \\
\hline \multicolumn{5}{|c|}{ TIA 500, degrees } \\
\hline Temporal & $8(8)$ & $18(8)$ & $10(7-14)$ & $<0.0001$ \\
\hline Nasal & $6(6)$ & $16(6)$ & $10(7-13)$ & $<0.0001$ \\
\hline \multicolumn{5}{|c|}{ TISA $500, \mu \mathrm{m}^{2}$} \\
\hline Temporal & $39(30)$ & $91(71)$ & $52(26-78)$ & 0.0003 \\
\hline Nasal & $23(24)$ & $65(30)$ & $42(29-16)$ & $<0.0001$ \\
\hline \multicolumn{5}{|l|}{ IT 500, $\mu \mathrm{m}$} \\
\hline Temporal & $453(97)$ & $414(62)$ & $40(1-78)$ & 0.042 \\
\hline Nasal & $453(63)^{\star *}$ & $393(73)^{\star *}$ & $61(30-92)$ & 0.0004 \\
\hline \multicolumn{5}{|l|}{ AOD750, $\mu \mathrm{m}$} \\
\hline Temporal & $145(103)$ & $247(115)$ & $102(58-146)$ & $<0.0001$ \\
\hline Nasal & $119(82)$ & $212(92)$ & $93(12-62)$ & $<0.0001$ \\
\hline \multicolumn{5}{|c|}{ TIA 750, degrees } \\
\hline Temporal & $10(7)$ & $16(8)$ & $6(3-9)$ & $<0.0001$ \\
\hline Nasal & $9(6)$ & $15(6)$ & $6(4-8)$ & $<0.0001$ \\
\hline \multicolumn{5}{|c|}{ TISA $750, \mu \mathrm{m}^{2}$} \\
\hline Temporal & $82(52)$ & $149(83)$ & $67(33-101)$ & $<0.0001$ \\
\hline Nasal & $64(39)$ & $116(43)$ & $51(32-71)$ & $<0.0001$ \\
\hline \multicolumn{5}{|l|}{ TICL, $\mu \mathrm{m}$} \\
\hline Temporal & $314(272)$ & 59 (139) & $255(158-350)$ & $<0.0001$ \\
\hline Nasal & 475 (196) & $84(13)$ & 391 (293-489) & $<0.0001$ \\
\hline \multicolumn{5}{|l|}{$\mathrm{MIBH}, \mu \mathrm{m}$} \\
\hline Temporal & $137(72)$ & $90(79)$ & $47(16-78)$ & 0.0046 \\
\hline Nasal & $150(77)$ & $89(77)$ & $61(34-88)$ & $<0.0001$ \\
\hline $\mathrm{ACD}, \mathrm{mm}$ & $2.15(0.34)$ & $2.15(0.30)$ & & 0.605 \\
\hline
\end{tabular}

Various parameters are measured by AS-OCT in dark conditions for both temporal and nasal angles. Differences are statistically significant if $\mathrm{p}<0.05$; 31 eyes of 31 patients, mean follow-up of 39 months, *paired t-test, **only 30 patients; Cl: Confidence interval 

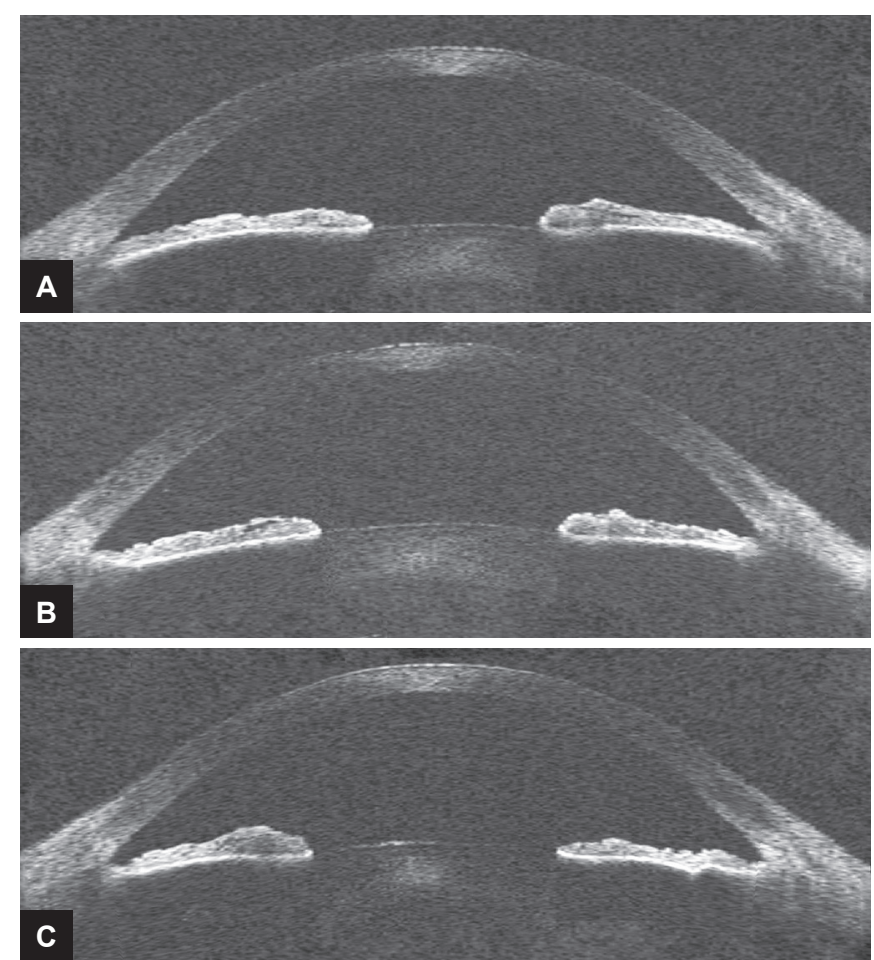

Figs 2A to C: (A) Preiridoplasty AS-OCT appearance; (B) Postiridoplasty AS-OCT appearance at 5 weeks; and (C) Postiridoplasty AS-OCT appearance at 29.5 weeks

of ITC on AS-OCT, but not to the extent that the eye could be considered completely nonoccludable (i.e., two residual quadrants of ITC on AS-OCT). Eighteen patients (58.1\%) fell within this category. "Failure" occurred when iridoplasty did not reduce the number of quadrants of ITC on AS-OCT and/or when cataract extraction was required to treat persistent occludable angles. Cataract surgery is a known treatment option for angle-closure glaucoma. ${ }^{26}$ In our cohort, four patients underwent cataract surgery during the follow-up period, with three $(3.12 \%)$ for persistent angle closure. Table 2 summarizes our success rates over the medium term.

From this cohort of patients, the AS-OCT temporal and nasal angle parameters such AOD, TIA, and TISA, measured at both 500 and $750 \mu \mathrm{m}$ from the scleral spur, showed a statistically significant increase 6 weeks after iridoplasty, and this was maintained over an average follow-up duration of 3 years. The IT 500 also showed a statistically significant reduction in peripheral IT in the medium term postiridoplasty.

Narayanaswamy et $\mathrm{al}^{27}$ in their randomized controlled trial (RCT) of 80 Asian patients with PAC or PACG also demonstrated similar increases of the indicators of the angle width, with AOD 500, AOD 750, TISA 750, and ARA 750 significantly increasing following iridoplasty at 1 year. However, the group went on to find that iridoplasty on its own was inferior to medical therapy in maintaining IOP of less than $21 \mathrm{~mm} \mathrm{Hg}$ at 1 year with an

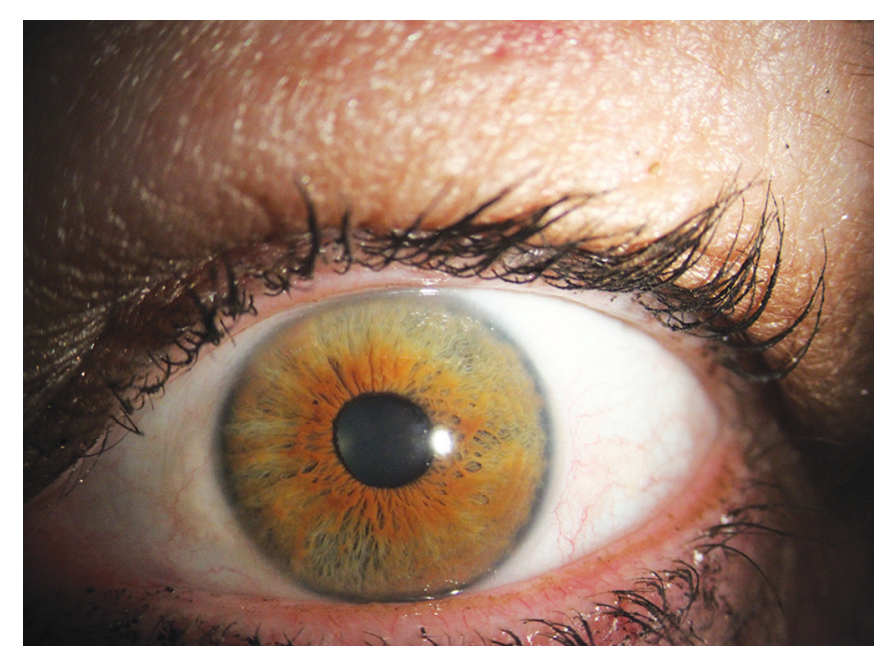

Fig. 3: Typical appearance of postiridoplasty scars

Table 2: Laser peripheral iridoplasty overall, complete, and partial success rate over the medium term

\begin{tabular}{llll}
\hline Time & Failure (\%) & $\begin{array}{l}\text { Complete } \\
\text { success }(\%)\end{array}$ & $\begin{array}{l}\text { Partial } \\
\text { success (\%) }\end{array}$ \\
\hline Initial follow-up 22.5 (7/31) & $45.1(14 / 31)$ & $32.3(10 / 31)$ \\
Final follow-up $16.1(5 / 31)$ & $25.8(8 / 31)$ & $58.1(18 / 31)$ \\
\hline
\end{tabular}

overall success rate of $70 \%$ using combined iridoplasty and medical therapy. Although our study did not specifically examine the effects of medical therapy, our cohort of patients maintained an IOP of 21 or less over the followup duration. A potential factor in the difference could be due to the patient population, with the RCT consisting of only Asians of predominantly Chinese background.

Iridoplasty has also been shown to reduce dependency on and reduce the number of antiglaucoma medications. In a prospective observation case-control study of 24 eyes in 12 patients, Ramakrishnan et $\mathrm{al}^{28}$ demonstrated a reduction by more than half in dependency and use of antiglaucoma medications at 1 year in patients who underwent iridoplasty.

Complications associated with iridoplasty are uncommon. One concern is the theoretical risk of corneal endothelial cell loss due to transfer of laser energy through the cornea. Müller et $\mathrm{al}^{29}$ could not demonstrate a significant difference in central endothelial cell density over an average of 30 months when comparing eyes that had undergone iridoplasty with the fellow untreated eye. Further, Narayanaswamy et $\mathrm{al}^{27}$ did not find any detrimental effect on the endothelial cell count or central corneal thickness at 1 year, while Ritch et $\mathrm{al}^{7}$ reported iridoplasty to be safe over a 6-year period with no complications from repeat treatment. Numerous other studies have also reported iridoplasty as safe with no significant associated complications. ${ }^{16,25,28}$ 
The limitations of our study include its retrospective design, single measure, and relatively small number of patients. We utilized scans only from the nasal and temporal quadrants due to the relative difficulty in identifying anatomical landmarks consistently in superior and inferior quadrants, a methodological issue that has previously been addressed in a similar fashion by other groups. ${ }^{23,30,31}$ It was also not possible to determine whether the AS-OCT scans imaged the exact same cross section of the angle before and after iridoplasty. We aimed to mitigate this by ensuring that all the AS-OCT images were centered on the pupil in the central horizontal meridian, in order to maximize scan consistency. A further limitation of AS-OCT is its inability to directly evaluate the ciliary body, such as for anterior rotation, which would have been of particular interest in this cohort of patients. Although this study utilized a single measurer, the scans being evaluated were fully masked, and the validity of the method already established as described above.

\section{CONCLUSION}

This study describes systematically the changes observed by AS-OCT in the ACA configuration induced by iridoplasty in the medium term in a cohort of patients with residual angle closure after iridotomy. Our data suggest that iridoplasty remains a useful adjunct in medium-term widening of the ACA. This can be particularly helpful in those with persistent angle closure after iridotomy but with no cataract. Iridoplasty can therefore, act as a temporizing measure to widen the drainage angle until such time that cataract surgery can be performed.

\section{REFERENCES}

1. Quigley HA, Broman AT. The number of people with glaucoma worldwide in 2010 and 2020. Br J Ophthalmol 2006 Mar;90(3):262-267.

2. Foster PJ, Johnson GJ. Glaucoma in China: how big is the problem? Br J Ophthalmol 2001 Nov;85(11):1277-1282.

3. Day AC, Baio G, Gazzard G, Bunce C, Azuara-Blanco A, Munoz B, Friedman DS, Foster PJ. The prevalence of primary angle closure glaucoma in European derived populations: a systematic review. Br J Ophthalmol 2012 Sep;96(9):1162-1167.

4. Foster PJ, Buhrmann R, Quigley HA, Johnson GJ. The definition and classification of glaucoma in prevalence surveys. $\mathrm{Br}$ J Ophthalmol 2002 Feb;86(2):238-242.

5. Thomas R, Walland MJ. Management algorithms for primary angle closure disease. Clin Exp Ophthalmol 2013 Apr;41(3):282-292.

6. He M, Friedman DS, Ge J, Huang W, Jin C, Lee PS, Khaw PT, Foster PJ. Laser peripheral iridotomy in primary angleclosure suspects: biometric and gonioscopic outcomes: the Liwan Eye Study. Ophthalmology 2007 Mar;114(3):494-500.

7. Ritch R, Tham CC, Lam DS. Long-term success of argon laser peripheral iridoplasty in the management of plateau iris syndrome. Ophthalmology 2004 Jan;111(1):104-108.
8. Ritch R, Tham CC, Lam DS. Argon Laser Peripheral Iridoplasty (ALPI): an update. Surv Ophthalmol 2007 May-Jun; 52(3):279-288.

9. Stevenson C, Leong JC, Ang GS, Wells AP. Spectral domain optical coherence tomography evidence on the mechanism of diode laser peripheral iridoplasty. Clin Experiment Ophthalmol 2015 Jul;43(5):474-476.

10. Weinreb, RN.; Friedman, DS. 3rd Global AIGS consensus meeting: angle closure glaucoma. Geneva: PS Printing (on behalf of Geneva Medical Publishers, Geneva, Switzerland); 2006. p. 112.

11. Friedman DS, He M. Anterior chamber angle assessment techniques. Surv Ophthalmol 2008 Jun;53(3):250-273.

12. Memarzadeh F, Li Y, Chopra V, Varma R, Francis BA, Huang D. Anterior segment optical coherence tomography for imaging the anterior chamber after laser peripheral iridotomy. Am J Ophthalmol 2007 May;143(5):877-879.

13. Müller M, Dahmen G, Pörksen E, Geerling G, Laqua H, Ziegler A, Hoerauf $\mathrm{H}$. Anterior chamber angle measurement with optical coherence tomography: intraobserver and interobserver variability. J Cataract Refract Surg 2006 Nov;32(11):1803-1808.

14. RadhakrishnanS,SeeJ,SmithSD,NolanWP,CeZ,FriedmanDS, Huang D, Li Y, Aung T, Chew PT. Reproducibility of anterior chamber angle measurements obtained with anterior segment optical coherence tomography. Invest Ophthalmol Vis Sci 2007 Aug;48(8):3683-3688.

15. Leung CK, Weinreb RN. Anterior chamber angle imaging with optical coherence tomography. Eye (Lond) 2011 Mar;25(3):261-267.

16. Yoong Leong JC, O'Connor J, Soon Ang G, Wells AP. Anterior segment optical coherence tomography changes to the anterior chamber angle in the short-term following laser peripheral iridoplasty. J Curr glaucoma Pract 2014 Jan-Apr;8(1):1-6.

17. Weinreb, R.; Friedman, D. Angle closure and angle closure glaucoma The Hague: Kugler Publications; 2006. p. 98.

18. Ang GS, Wells AP. Changes in Caucasian eyes after laser peripheral iridotomy: an anterior segment optical coherence tomography study. Clin Exp Ophthalmol 2010 Nov;38(8): 778-785.

19. Pavlin CJ, Harasiewicz K, Foster FS. Ultrasound biomicroscopy of anterior segment structures in normal and glaucomatous eyes. Am J Ophthalmol 1992 Apr;113(4):381-389.

20. Radhakrishnan S, Goldsmith J, Huang D, Westphal V, Dueker DK, Rollins AM, Izatt JA, Smith SD. Comparison of optical coherence tomography and ultrasound biomicroscopy for detection of narrow anterior chamber angles. Arch Ophthalmol 2005 Aug;123(8):1053-1059.

21. He M, Friedman DS, Ge J, Huang W, Jin C, Cai X, Khaw PT, Foster PJ. Laser peripheral iridotomy in eyes with narrow drainage angles: ultrasound biomicroscopy outcomes. The Liwan Eye Study. Ophthalmology 2007 Aug;114(8): 1513-1519.

22. Woo EK, Pavlin CJ, Slomovic A, Taback N, Buys YM. Ultrasound biomicroscopic quantitative analysis of light-dark changes associated with pupillary block. Am J Ophthalmol 1999 Jan;127(1):43-47.

23. How AC, Baskaran M, Kumar RS, He M, Foster PJ, Lavanya R, Wong HT, Chew PT, Friedman DS, Aung T. Changes in anterior segment morphology after laser peripheral iridotomy: an anterior segment optical coherence tomography study. Ophthalmology 2012 Jul;119(7):1383-1387. 
24. Lee KS, Sung KR, Shon K, Sun JH, Lee JR. Longitudinal changes in anterior segment parameters after laser peripheral iridotomy assessed by anterior segment optical coherence tomography. Investig Ophthalmol Vis Sci 2013 May;54(5):3166-3170.

25. Sng CC, Aquino MC, Liao J, Zheng C, Ang M, Chew PT. Anterior segment morphology after acute primary angle closure treatment: a randomised study comparing iridoplasty and medical therapy. Br J Ophthalmol 2016 Apr;100(4):542-548.

26. Azuara-Blanco A, Burr J, Ramsay C, Cooper D, Foster PJ, Friedman DS, Scotland G, Javanbakht M, Cochrane C, Norrie J, EAGLE study group. Effectiveness of early lens extraction for the treatment of primary angle-closure glaucoma (EAGLE): a randomised controlled trial. Lancet 2016 Oct;388(10052):1389-1397.

27. Narayanaswamy A, Baskaran M, Perera SA, Nongpiur ME, Htoon HM, Tun TA, Wong TT, Goh D, Su DH, Chew PT, et al. Argon laser peripheral iridoplasty for primary angle-closure glaucoma: a randomized controlled trial. Ophthalmology 2016 Mar;123(3):514-521.
28. Ramakrishnan R, Mitra A, Abdul Kader M, Das S. To study the efficacy of laser peripheral iridoplasty in the treatment of eyes with primary angle closure and plateau iris syndrome, unresponsive to laser peripheral iridotomy, using anterior-segment OCT as a tool. J Glaucoma 2016 May;25(5): 440-446.

29. Müller L, Reeves GM, Leong JC, Wells AP. How safe is diode laser peripheral iridoplasty for the corneal endothelium? Clin Exp Ophthalmol 2016 Nov;44(8):735-737.

30. Day AC, Garway-Heath DF, Broadway DC, Jiang Y, Hayat S, Dalzell N, Khaw KT, Foster PJ. Spectral domain optical coherence tomography imaging of the aqueous outflow structures in normal participants of the EPIC-Norfolk Eye Study. Br J Ophthalmol 2013 Feb;97(2):189-195.

31. Nongpiur ME, Haaland BA, Friedman DS, Perera SA, He M, Foo LL, Baskaran M, Sakata LM, Wong TY, Aung T. Classification algorithms based on anterior segment optical coherence tomography measurements for detection of angle closure. Ophthalmology 2013 Jan;120(1):48-54. 